\title{
Questes
}

\section{Le Northumberland: les attributs d'une terre de la marge dans quelques textes arthuriens}

Sophie Albert

\section{OpenEdition}

\section{Journals}

Édition électronique

URL : http://journals.openedition.org/questes/2226

DOI : 10.4000/questes. 2226

ISSN : 2109-9472

\section{Éditeur}

Les Amis de Questes

\section{Édition imprimée}

Date de publication : 15 juin 2006

Pagination : 40-51

ISSN : 2102-7188

\section{Référence électronique}

Sophie Albert, «Le Northumberland : les attributs d'une terre de la marge dans quelques textes arthuriens », Questes [En ligne], 9 | 2006, mis en ligne le 01 janvier 2014, consulté le 30 avril 2019. URL : http://journals.openedition.org/questes/2226 ; DOI : 10.4000/questes.2226 


\title{
Le Northumberland : les attributs d'une terre de la marge dans quelques textes arthuriens
}

\author{
Sophie ALBERT
}

Comme l'a montré Cedric E. Pickford', le Hombre fait l'objet d'un traitement particulier dans un certain nombre de textes arthuriens: dans le Lancelot en prose, le fleuve, entouré de rochers, est le lieu où s'élève la Douloureuse Garde, château dont Lancelot résoudra les coutumes; c'est également le lieu où réside le géant Caradoc, seigneur de la Douloureuse Tour, à laquelle conduit un pont de fer. Le Hombre, dans ce roman, est présenté comme une variante du fleuve qui, dans le Chevalier de la Charrette, mène au royaume de Gorre - une variante, plus largement, de tous les cours d'eau qui protègent l'accès à d'étranges royaumes. Cette sombre représentation du Hombre est perceptible dès le Roman de Brut de Wace, translation en français de l'Historia Regum Britanniae. Chez Geoffroy, le Hombre apparaît dans un passage qui accumule les légendes étiologiques : de même que Locrinus donne son nom au royaume de Logres, Kamber à la Cambrie, etc., de même Humber, le roi des Huns, donne son nom au fleuve Hombre. L'évocation est amplifiée dans le Roman de Brut: Wace développe le portrait de Humber, un «cruels huem » (v. 1300), «Uns hoem forment par mer cremuz,/ Ki les illes alout guastant/ E les rivages tuz robant $»^{2}$. Humber devient, en somme, un véritable faiseur de terres

\footnotetext{
${ }^{1}$ PiCKFORD, Cedric Edward, « The river Humber in French Arthurian Romances », dans The legend of Arthur in the Middle Ages, Studies presented to A. H. Diverres by colleagues, pupils and friends, P. B. Grout, R. A. Lodge, C. E. Pickford, E. K. VARTY (éds.), Woodbridge, Suffolk, Boydell and Brewer, 1983 (Arthurian Studies, VII), pp. 149-159.

${ }^{2}$ WACE, Le Roman de Brut, Ivor ARNOLD (éd.), Paris, 1938-1940, v. 1294-96.
} 
gastes. Quelques vers plus loin, Wace insiste, bien plus que ne le faisait Geoffroy, sur l'homonymie entre le personnage et le fleuve ${ }^{3}$. Le Hombre est donc explicitement placé sous un parrainage peu recommandable.

Etant donné, d'une part, cette origine onomastique détestable et, d'autre part, la tendance de l'imaginaire médiéval à rejeter aux marges du monde connu les manifestations de l'altérité, il ne serait pas anormal que le Northumberland, terre située au-delà du Hombre, soit doté de traits inquiétants ${ }^{4}$. Le but de cet exposé sera de préciser quelques-uns des contenus par lesquels les auteurs de romans arthuriens ont représenté le caractère proprement marginal de ce royaume ${ }^{5}$ et, notamment, à quels types de personnages et de comportements « sociaux » correspond cette frange géographique.

Le Northumberland est totalement absent de l'œuvre de Chrétien de Troyes : 1'action de ses romans, circonscrite dans le temps, l'est aussi, si l'on excepte Cligès, dans l'espace. Pour que les romans arthuriens s'ouvrent à un espace géographique « réaliste » dépassant les frontières du royaume de Logres, il fallait parallèlement qu'ils renouent avec le cadre temporel des chroniques ; ce lien, les romans en prose l'établissent, en particulier le cycle du Lancelot-Graal. En même temps que le temps du roman s'étend au-delà des douze années de paix dans lesquelles s'inscrivaient les romans de Chrétien, l'espace s'élargit, incluant, outre l'Orient des origines, plusieurs pays d'Europe occidentale. Le Northumberland fait ainsi une nouvelle entrée dans la littérature arthurienne.

${ }^{3}$ « Pur Humbro fu Humbre apelee ;/Humbre cest non l'eue reçut/Pur Humbro que dedenz morut » (ibid., v. 1312-1314).

${ }^{4}$ Cette hypothèse ne vaut pas pour tous les romans arthuriens : le relevé de toutes les occurrences du Northumberland dans le Tristan en prose montre que ce royaume y est un pays «comme les autres ». Ce seront dans ce roman d'autres royaume (le Sorelois par exemple) qui recevront un caractère « étrange ».

${ }^{5}$ La brève étude qui suit se borne à poser quelques jalons sur les représentations du Northumberland, et n'embrasse évidemment pas la totalité de la littérature arthurienne. Elle constitue une première ébauche d'un travail que j'aimerais développer plus tard. 
Reste à savoir dans quelle mesure, et sous quelle forme, les écrivains transposent ou adaptent les données du Roman de Brut de Wace.

Dans le Merlin en prose, au cours d'un dialogue célèbre entre Merlin et Blaise, le prophète commande à son « scribe » de se retirer en Northumberland :

... et demenderas une terre qui a non Norhombellande; et cele terre si est plene de molt granz forez et si est molt estrange a genz dou païs meimes, que il i a de tels parties ou nus n'a encor esté ${ }^{6}$.

Territoire encore inhabité, couvert de bois immenses : c'est avant tout l'image d'une terre inculte qui domine, aux franges de la civilisation. Arthur, quand le prophète disparaît, le cherche en premier lieu dans les forêts de ce pays. Or Merlin, depuis la Vita Merlini, emprunte certains de ses attributs à la figure de l'homme sauvage ; qu'il vive, une partie du temps au moins, dans les bois du Northumberland, n'est pas sans évoquer les affinités qu'il entretient avec cette figure.

Dans le Lancelot propre, le Northumberland occupe une place minime: une dizaine d'occurrences dans l'ensemble de l'œuvre, pourtant considérable. L'une de ces mentions, parmi les plus significatives, intervient lors du premier séjour en Sorelois de Galehaut et Lancelot.

Ce dit li contes que antre Galehot et son conpaignon errerent par lor jornees, que il vint en la terre dom il estoit sires. Ce fu la terre de Sorolois qui siet antre Gales et les Estranges Illes. Icele terre n'estoit mies Galehot d'ancesserie, ainz l'avoit gaaigniee par force sor lo roi Gloier, un neveu au roi [de] Northunberlande. [Galehaut garde la fille de ce Gloier], si la devoit doner a fame a un sien neveu qui ores estoit mout petiz, si li avoit tote la terre de Sorelois otroiee a l'ore que il seroit chevaliers ${ }^{7}$.

Comme 1'a montré Emmanuèle Baumgartner, le royaume de Sorelois, « entièrement entouré d'eau, isolé et préservé par deux 'passages assés felon et

\footnotetext{
${ }^{6}$ Robert De Boron, Merlin, roman du XIII ${ }^{e}$ siècle, MichA, Alexandre (éd.), Genève, Droz, $2000, \S 23$.

${ }^{7}$ Lancelot du Lac II, KenNeDY, Elspeth (éd.), CHÊNERIE, Marie-Luce (trad.), Paris, Librairie Générale Française, 1993, p. 28.
} 
orguellous' (VIII 129) que défend en permanence une garnison nombreuse », est décrit dans le Lancelot comme un double du royaume de Gorre ${ }^{8}$. Sa localisation « antre Gales et les Estranges Illes » le situe entre un pays qui relève d'une géographie « réaliste » et une contrée merveilleuse. Dans ce chassé-croisé de lieux et de personnages, une cohérence se fait jour. Tous les personnages liés au Sorelois participent, d'une manière ou d'une autre, d'une forme d'altérité : Galehaut par sa parenté avec la Belle Géante et par l'ancrage géographique de son fief, les Etranges Iles ; Gloier par sa parenté avec le roi de Northumberland, terre des confins, et par la forme de son royaume, le Sorelois, enclos par une mauvaise coutume. Dans ce monde, on reste entre soi : Galehaut confiera un temps la garde du Sorelois à Baudemagu, le père de Méléagant; il compte marier la fille de Gloier avec son propre neveu, comme pour unir deux lignées marquées par une même ambivalence.

Quelques années plus tard, la Suite $d u$ Merlin offre une notation intéressante :

$\S 321$. La roine Elainne, si tost comme elle connut la damoisele de Norhomberlande, elle li fist joie et feste mervilleuse. Ne ne cuidiés pas, entre vous qui oés ces contes, que chis Norhomberlande dont je parole soit li roiames de Norhomberlande qui estoit entre le roiaume de Logres et chelui de Gorre : che seroit folie a cuidier, car chis Norhomberlande estoit en la Petite Bretaigne et li autres Norhomberlande en la Grant ${ }^{9}$.

La localisation géographique proposée pour le Northumberland de GrandeBretagne, « entre le roiaume de Logres et chelui de Gorre », rappelle fortement celle que le Lancelot attribuait au Sorelois : les deux contrées sont placées à michemin entre une terre «bonne » et une terre «mauvaise », cette configuration

${ }^{8}$ BAumgartner, Emmanuèle «Géants et chevaliers », The spirit of the Court. Selected proceedings of the Fourth Congress of the International Courtly Literature Society (Toronto 1983), Burgess, Glyn S. et TAYlor, Robert A. (éds.), Cambridge, D.S. Brewer, 1985, p. 9$22:$ p. 12.

${ }^{9}$ La Suite du Roman de Merlin, Roussineau, Gilles (éd.), Genève, Droz, 1996, § 321. La « damoisele de Norhomberlande » est la dame du Lac, dont le père habite ce royaume. 
renvoyant à leur position axiologique ambiguë. D'autre part, l'auteur de la Suite invente, à côté du premier Northumberland - qui était dans le Merlin la terre de prédilection du mage -, un second Northumberland, terre d'origine de la future Dame du Lac. Le passage intervient juste avant que celle-ci n'entombe Merlin, mettant fin à sa carrière officielle de prophète, pour se substituer à lui dans ses fonctions d'adjuvant de la cour. Le transfert de pouvoir d'un enchanteur à l'autre passe par l'invention d'un nouveau Northumberland, destiné à montrer l'égale dignité des deux mages. En même temps, l'ancrage continental de ce nouveau royaume s'accorde avec le futur champ d'action de la fée, qui élèvera Lancelot en Gaule et non dans les îles britanniques.

Ainsi, dans le Lancelot et la Suite Merlin, le Northumberland de GrandeBretagne est intégré à un réseau de royaumes étranges, au sein duquel il occupe une place intermédiaire : lié au Sorelois par ses souverains successifs, il se situe entre la terre normative de Logres et le redoutable royaume de Gorre; si on rapporte sa position au système masculin du Lancelot tel que l'a défini JeanRené Valette ${ }^{10}$, il serait plutôt du côté de Galehaut que de Méléagant - du côté des grands chevaliers, mais pas des chevaliers mauvais. Cette forme d'altérité géographique diffère sensiblement de l'image que convoquait le Roman de Merlin, celle d'une forêt "sauvage », étrangère à l'univers domestique. Le Merlin trouve toutefois un prolongement dans la Suite, dans la mesure où les deux romans associent, au Northumberland ou à un homonyme gaulois inventé pour la circonstance, un personnage pourvu de savoirs magiques.

Face à ces textes qui positionnent le Northumberland par rapport à une «merveille»- que celle-ci soit un royaume étrange ou un personnage merveilleux, fée, enchanteur ou grand chevalier -, Guiron le Courtois construit

10 Valette, Jean-René, La Poétique du merveilleux dans le Lancelot en prose, Paris, Champion, 1998. 
la marginalité de ce royaume à partir d'éléments purement rationnels ${ }^{11}$. Le Northumberland se présente à première vue comme un double du royaume d'Arthur; ses cours, orchestrées par des rois puissants, manifestent une richesse égale à celle de la cour de Logres, ses tournois attirent toute la chevalerie. Le premier décalage avec le royaume de Logres est d'ordre temporel: le Northumberland est généralement mentionné dans des récits enchâssés, ce qui a pour effet immédiat de l'associer à un temps « autre ». Aussi, relativement peu présent dans le « roman de Meliadus », il prend dans le « roman de Guiron » une importance remarquable ${ }^{12}$ : sur la quarantaine de récits enchâssés de ce roman, dix font intervenir le Northumberland.

Les habitants de ce pays correspondent d'abord à des types. Type de la traîtresse : plusieurs récits racontent comment un chevalier, toujours plein de bonne foi, se fait abuser par une perfide demoiselle. Dans trois cas $(\S 56,98$, 112-115), celle-ci est originaire du Northumberland; Meliadus, narrateur du $\S 56$, précise même que sa demoiselle a un accent très prononcé ${ }^{13}$. Le $\S 98$ reprend le thème de la femme de Putiphar: la dame du Northumberland, amoureuse d'un ami de son époux, se fait éconduire et, pour se venger, accuse le trop honnête chevalier d'avoir tenté de la violer. Quant à la fille du roi de Northumberland, elle cherche par tous les moyens à faire mourir Febus,

11 En l'absence d'édition intégrale, on se reportera à l'ouvrage de LATHUILLERE, Roger, " Guiron le Courtois » : étude de la tradition manuscrite et analyse critique, Genève, Droz, 1966, noté ici «analyse », qui donne un résumé de tous les manuscrits connus en 1966. Les numéros de paragraphes renvoient à cet ouvrage.

${ }^{12}$ Le « roman de Meliadus » occupe les $§ 1-51$ de l'analyse de Lathuillère, le « roman de Guiron » les §52-130. Les deux textes ont circulé de manière autonome avant d'être rassemblés dans des manuscrits uniques. Ainsi, plusieurs manuscrits ne contiennent que le « roman de Meliadus » (par exemple L1 et V2, tous deux du XIV siècle; n. a. f. 5243, XIV siècle), tandis que deux témoins parmi les plus anciens ne donnent que le «roman de Guiron » (Marseille, BM, 1106, et Privas, Archives départementales de l'Ardèche, $\mathrm{n}^{\circ}$ I (F7), tous deux datés de la fin du XIII ${ }^{\mathrm{e}}$ siècle). Dans le ms. de Paris, BNF, fr. 350, le « roman de Guiron » est séparé du « roman de Meliadus » par un blanc d'un folio et demi.

13 On peut lire ce récit dans TRACHSLER, Richard (dir.), "Guiron le Courtois ». Une anthologie, textes édités, traduits et présentés par ALBERT, Sophie, PlaUT, Mathilde et Plumet, Frédérique, Alessandria, Edizioni dell’Orso, 2004, pp. 285-313. 
chevalier admirable, alors même que celui-ci multiplie les gestes d'allégeance $(\S 112-115)^{14}$.

Second type, plus fugitif puisqu'il n'apparait que dans un seul passage, celui du chevalier ravisseur. Au $\S 54$, Gauvain relate comment un chevalier aux armes vermeilles fit naguère irruption à la cour d'Arthur, embrocha deux ou trois chevaliers et emporta une demoiselle. Or ce chevalier vermeil était un chevalier de Northumberland prénommé Heliadel. Plus tard, Gauvain, dans le récit principal cette fois, rencontre à nouveau Heliadel, qui poursuit de sa haine une demoiselle solitaire. Outre qu'il illustre manifestement le type du chevalier ou du géant ravisseur, le personnage d'Heliadel est peut-être inspiré de Brehus sans Pitié, le célèbre ennemi des dames. En effet, comme Brehus, il surgit inopinément et se définit avant tout par sa haine pour la gent féminine.

Enfin, plusieurs récits enchâssés du «roman de Guiron » présentent une image du mauvais gouvernement et, en particulier, du mauvais roi. Ils obéissent à un schéma assez stable: un chevalier arrive à la cour du roi de Northumberland ou, dans un cas, de son frère. Il est calomnié $(\S 56,81)$ ou, souvent, pris pour un lâche $(\S 56,62,96)$, soit qu'il ait échangé ses armes avec un couard ( $(96)$, soit qu'on lui ait volé les siennes ( $(56)$. Dans tous les cas, le roi de Northumberland préside à un jugement inique, prenant la calomnie pour véridique ( $(81)$ ou décidant de châtier le faux couard $(\S 56,96)$. Le héros ainsi abusé reçoit à la fin du récit une honte cuisante, à laquelle participe la cour entière. Dans ces récits, l'image des rois de Northumberland s'oppose à la représentation du roi idéal, apte à rendre justice.

Plus radicalement encore, le récit des $§ 112-115$ montre un roi de Northumberland soumis à un tribut que deux géants imposent à toute sa contrée. Loin de s'en plaindre, le roi est ravi de voir les géants percevoir le tribut, et le narrateur explique que ce dernier résulte d'un accord : contre la vie du roi, sauvé

${ }^{14}$ Pour une édition récente du passage, voir ibid., pp. 87-149. 
autrefois par les géants, les habitants du royaume ont juré de payer chaque année aux géants un tribut humain et matériel. Au demeurant, les géants et le roi, réunis à l'occasion d'une fête païenne, font preuve de la plus cordiale entente, multipliant les politesses et les démonstrations d'amitié. Cette alliance entre un souverain de Northumberland et des géants ainsi que leur commune adhésion à des croyances païennes signalent la spécificité sociale et religieuse du royaume.

Les différentes incarnations du type du mauvais roi, qui comportent toutes une dimension «socio-juridique », entrent dans un système de représentation plus large, impliquant une hiérarchisation entre les comportements des différents acteurs du « roman de Guiron ». Au sein de ce système, le Northumberland est le théâtre de pratiques sociales contestables, fondamentalement en désaccord avec les pratiques normatives de la «bonne» chevalerie - les chevaliers d'Arthur.

Ce trait est confirmé, en premier lieu, par plusieurs détails ayant trait aux systèmes de parenté. Dans le récit du $§ 96$, le narrateur précise qu'Aquilan, frère du roi de Northumberland, a quatorze fils, dont cinq bâtards. Les fratries du « roman de Guiron » comptent rarement plus de quatre chevaliers; une fratrie aussi nombreuse fait exception ${ }^{15}$. D'autre part, il est non moins exceptionnel que l'on signale la présence de bâtards. Un autre épisode dénote une relative anomalie : au $\S 101$, les fils du roi de Northumberland se disputent l'héritage de

${ }^{15}$ Seul autre cas du «roman de Guiron » où une fratrie dépasse la douzaine, le $\S 128$ : un ermite raconte à Danain que naguère, Lyas et Helyon, deux seigneurs ayant respectivement quinze filles et quinze fils, s'entretuèrent; sur son lit de mort, chacun d'eux fit promettre à sa progéniture de ne pas se marier tant que subsisterait un seul membre du clan adverse. Une fois les deux pères enterrés, les fils d'Helyon assaillent les filles de Lyas, qui demandent son aide à Galehaut le Brun. Celui-ci repousse les fils d'Helyon et institue une coutume qui réaffirme le serment que les enfants ont juré à leur père : Galehaut "confrema des damoiseles ce que Lÿas en avoit fait : nule ne se puet marier tant que nus des freres vive ». Il ferme ensuite la vallée où vivent les enfants de Lyas et d'Helyon par un pont qui se relève une fois franchi, interdisant à quiconque de rebrousser chemin. Les filles de Lyas sont ainsi emprisonnées dans un nouveau Val sans Retour. La création de la coutume permet d'enclore deux lignages anormaux, stérilisés d'abord par le refus du mariage. 
leur père ; chacun prend un champion pour défendre sa cause, avant que le roi Uterpandragon ne résolve l'affaire par un accord à l'amiable. Le motif des frères ennemis, repris par de nombreux romanciers après les sœurs de Noire Epine du Chevalier au Lion, concerne, comme par hasard, deux princes du Northumberland.

En second lieu, plusieurs personnages du Northumberland mettent en péril le code implicite qui régit le système vindicatoire du roman ${ }^{16}$. Au $\S 25^{17}$, un hôte relate au Chevalier sans Peur une histoire de vengeance qui opposa jadis son lignage, composé d'habitants du Northumberland, à Meliadus de Leonois. Ce dernier avait tué Delis, un membre du lignage du narrateur ; les parents du mort assaillirent Meliadus à vingt-deux contre un, mais le héros se défendit et triompha sans peine de la troupe. Les parents du mort formèrent alors une expédition punitive de quatre membres qui, ayant surpris Meliadus dans son sommeil, l'attaqua et le blessa gravement. Les parents de Delis contreviennent doublement aux lois des vengeances romanesques : en se mettant à vingt-deux contre un, quand un chevalier digne de ce nom se venge seul des offenses qu'on lui a infligées; en usant de traîtrise, parce qu'ils attaquent Meliadus dans son sommeil. Si leur désir de vengeance est légitime, leurs procédés ne le sont pas. Heureusement, le héros est sauvé par l'intervention du narrateur, indigné par l'attitude de ses proches.

Le récit du $\S 77$ met en scène un autre type de démesure. Le roi du Northumberland rassemble une armée contre le seigneur de l'Etroite Marche :

Li rois ot assemblé trop grant host et grant force de gent que de sis parent, que de sis amis, que de sis homes lÿges; et pour ce que en la terre del segnour de l'Estroite Marche estoit mort celui an un fill del roi de Noubellande et li rois cuidoit bien vraiement que li sires de l'Estroite Marche l'eüst mort assambla si grant host que ce

16 L'étude de ce système vindicatoire fait l'objet d'un chapitre entier de ma thèse ; je m'appuie ici sur des éléments que ce chapitre a mis en évidence.

${ }^{17}$ Une fois n'est pas coutume, ce récit enchâssé se situe dans le « roman de Meliadus ». 
estoit merveille del veoir : la gent venoient de toute part, la force i croist en moult de guises. (ms. de Paris, BNF fr. 350, fo 193d)

L'usage du verbe "cuidoit» est riche d'enseignements, ce verbe étant systématiquement réservé, dans le «roman de Guiron», aux croyances erronées : les soupçons du roi de Northumberland, très manifestement, ont peu de fondement. Sa vengeance, privée de mobile valable, est mise en échec par Hector le Brun, héros insurpassable, qui repousse la troupe des assaillants.

Dans les deux cas, les gens du Northumberland ne parviennent pas à assouvir leur désir de vengeance. Cette non réalisation de la vengeance, dans le système vindicatoire de Guiron le Courtois, est le meilleur signe de son absence de légitimité : lorsqu'un «bon » chevalier se venge d'un géant ou d'un chevalier des confins, il réussit toujours à accomplir son devoir de vengeance ; à l'inverse, lorsqu'un géant ou un chevalier des confins cherche à se venger d'un héros, il échoue immanquablement. La place des habitants du Northumberland au sein du système vindicatoire du roman traduit l'ambivalence de leur position axiologique, plus proche de celle des géants que de celle des «bons» chevaliers.

Le Northumberland, terre de la marge, reçoit donc, au fil des textes, plusieurs qualifications susceptibles de donner un contenu à sa marginalité. Face à des représentations qui engagent un arrière-plan merveilleux, Guiron le Courtois propose des modes de mise à la marge plus rationnels, qui touchent aux pratiques sociales et à la valeur morale des habitants de ce royaume. Aux forêts du Merlin, l'auteur a substitué une cour régalienne ; à un monde inculte et sauvage, un univers possédant ses propres lois, reflets dégradés de celles de la cour arthurienne. La nature des normes transgressées par les gens du Northumberland, essentiellement sociales et juridiques, est significative des préoccupations du «roman de Guiron », qui manifeste un intérêt certain pour la 
représentation littéraire des codes sociaux et des systèmes de parenté : chaque écrivain construit ses marges en fonction d'un univers romanesque spécifique.

\title{
En guise d'épilogue...
}

A la fin du XIV ${ }^{\mathrm{e}}$ siècle, quand Jean Froissart écrit le roman Meliador ${ }^{18}$, il reprend, des romans arthuriens, l'idée générale du Northumberland comme terre «autre ». Mais cette fois, ce pays devient l'une des terres privilégiées de l'aventure chevaleresque, refuge de tous les périls. Agamanor y trouve « mainte aventure grande » (v. 13902) que le narrateur se garde de conter $^{19}$; même prétérition à propos de la chevauchée de Dagor :

\author{
S'en chevauce Dagors li frans \\ Et entre ens [en] Norchombrelande : \\ C'est une contrée moult grande ; \\ S'adont le fu, elle est encore. \\ Ançois qu'on puist venir au cor, \\ Il y a tamainte montagne \\ Et tamainte aventure estragne, \\ Des queles ores me tairai, \\ Et d'autre cose parlerai. (v. 28102-28110)
}

Le Northumberland revêt ainsi une géographie particulière (des montagnes, à rapprocher des forêts du Merlin) et une aura légèrement inquiétante. Parallèlement, le fleuve Hombre se charge à nouveau d'une coloration merveilleuse qui s'était beaucoup estompée dans les romans en prose :

[Sagremor chevauche]

.I. jour s'en vint li amoureus,

Chevaucant parmi une lande

Et entre en la forest moult grande,

Qui de lonch tenoit .II. journées.

18 JEAN Froissart, Méliador. Roman comprenant les poésies lyriques de Wenceslas de Bohême, duc de Luxembourg et de Brabant, Longnon, Auguste (éd.), Paris, Firmin Didot, 3 tomes, 1895-1899.

19 «Des queles ne puis par raison/Toutes parler », v. 13904-13905. 
En celle forest y ot fées,

Des queles je vous parlerai.

La forest ot nom Archenai.

De la muet, de desous .I. ombre,

La grosse riviere dou Hombre.

Moult y faisoit adont divers, mais li temps est or au revers,

Car pays y a habitable,

Bien plaisant et moult pourfitable.

Ceste forest dessus nommée,

Qui lors estoit moult renommée

D'aventures moult merveilleuses

Et as pluiseurs tres perilleuses,

Estoit moult belle et moult ombrue. (v. 28362-28379)

La forêt des fées, très « ombrue », jouxte le Hombre, dans un passage dont les jeux de mots rappellent ceux du Roman de Brut. Meliador confère toutefois une histoire à ce paysage : autrefois riche en aventures, le Northumberland est à son époque conquis, civilisé, non plus inculte, mais «habitable» et « pourfitable » : la marge a été intégrée, définitivement, au monde domestique. 